\title{
Twice Flipped - Avoiding Milling Curtains in FIB Prepared TEM Specimens of Nano-Electronics Devices.
}

\author{
Jerzy Gazda, Jorge Duarte, and Fawn Daby-Merrill - Cerium Laboratories LLC, 5204 E. Ben White
} Blvd. MS-512, Austin, TX 78741

We present a method of preparation of transmission electron microscopy (TEM) specimens of nano-electronic devices with Focused Ion Beam (FIB) tools that significantly reduces specimen thickness variation over the area of interest. The general methodology is a combination of and an extension to Micro-Sampling FIB specimen preparation technique described by T. Kamino et. al. [1] and the back-side milling method [2].

An example of milling curtains obscuring the interface between tungsten filled contact and Si-substrate in a Flash memory device is shown in Figure 1. Our method re-directs ion flow to avoid formation of curtains in the target area. In this example, we use FEI Strata-400DB-FIB equipped with FlipStage ${ }^{\mathrm{TM}}$ and Omniprober200 ${ }^{\mathrm{TM}}$ as efficient means of accomplishing specimen rotation. However we envision that the general idea behind this method can be adapted to various FIB/micromanipulator platforms and to a bulk specimen preparation from a multi-phase material where there exist vast density variations that lead to formation of milling curtains. The essence of the method dictates preparation of the specimen from a direction that allows direct access to the feature of interest through uniform material and avoids milling through tough materials above.

Without further ado, the method starts by routine preparation steps to produce targeted specimen of the feature of interest. In case of nano-electronics devices, stage navigation with design CAD layout, or defect review maps can be used to locate the target. The scripting capabilities of modern FIBs allow rapid and unassisted preparation of specimens up to the step of specimen extraction from the wafer/device. In case that a sub-stage and in-situ micromanipulator is available, the preparations steps to attach the lamella to probe and to remove it from the substrate are performed. Specimen is then attached to the grid, or any other suitable temporary support, that can be tilted to facilitate the inversion (flips.) In case of FEI-FlipStage ${ }^{\mathrm{TM}}$ tools, the specimen is first attached to the grid positioned parallel to the surface of the wafer/device but perpendicular to the traditional mounting for cross-sections. Specimen is then released from micromanipulator. An example of the arrangement is shown in Figure 2. This corresponds to the position described as $90^{\circ}$ in the tool's software that is usually used for STEM observation. The grid is then brought to $0^{\circ}$ or to the traditional specimen preparation orientation. At this point, the specimen is reattached to micromanipulator again, and then released from the supporting grid (as shown in Figure 3) and the grid is again lowered to $90^{\circ}$ position. Subsequent re-attachment to the grid places the specimen with Si-substrate above the area of interest. At that time, the grid is raised to $0^{\circ}$ and the remaining preparation steps are conducted to produce electron transparent lamella. An example of completed specimen is shown in STEM micrograph at low magnification in Figure 4 and in TEM micrograph at moderate magnification in Figure 5. In combination with lowered accelerating voltage ion beam milling this procedure allows preparation of ultrathin sections with AOIs on the order of $<50 \mathrm{~nm}$ thickness and lack of sample thickness variation under the contacts/vias. Figure 6 shows example of higher magnification TEM micrograph showing clear interface between silicon substrate, Ti-silicide reaction layer, the multi-layer diffusion barrier, and a tungsten filled contact.

In conclusion, the described method reduces operator's frustration arising from requirement for multiple re-prep to observe in the highest detail critical layers in nano-sized semiconductor devices otherwise obscured by artifacts of focused Gallium ion beam milling in the FIB.

References:

1) T. Kamino et. al. in "Introduction to FIB Instrumentation, Theory, Techniques, and Practice" ed. by. L. Giannuzzi and F. A. Stevie, Springer, New York, 2005, p. 237

2) Stephen M. Schwarz et.al., Microscopy and Microanalysis, 9 (Suppl. 2), 2003, p. 116 


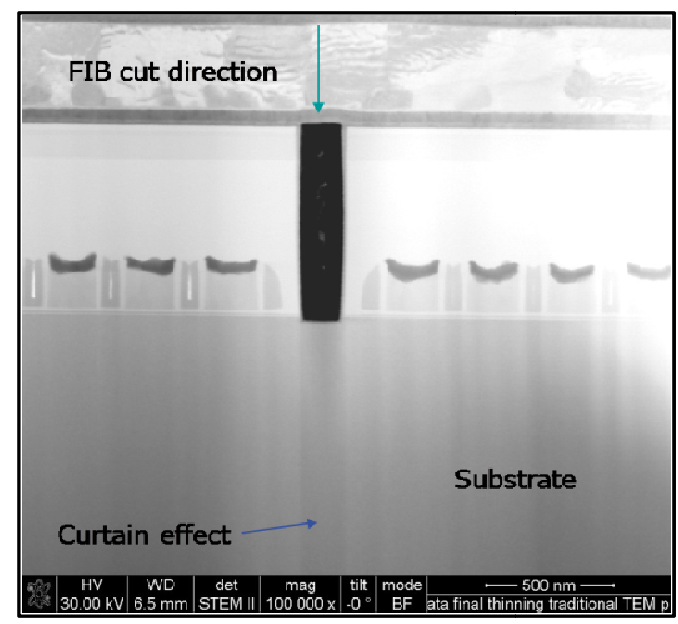

Figure 1. Milling curtains on traditional FIB prepared specimens due to variation in removal rate of materials.

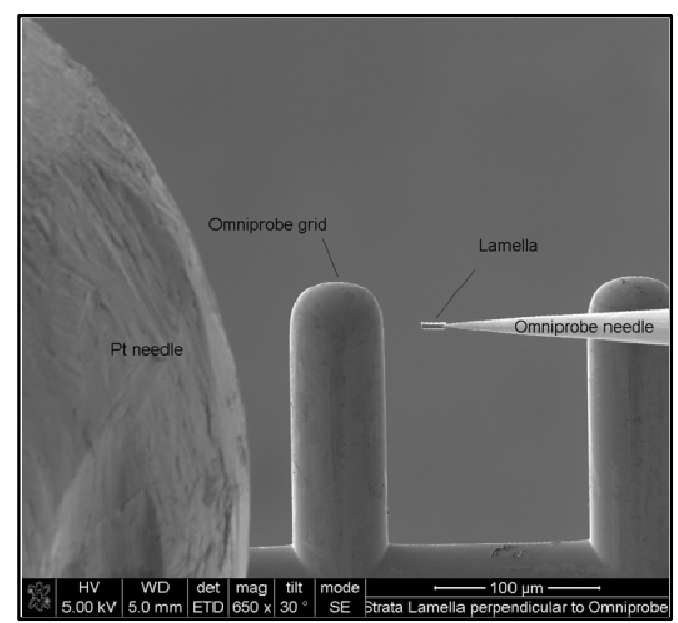

Figure 2. Specimen near a grid (positioned at " $90^{\circ}$ ") mounted parallel to wafer surface.

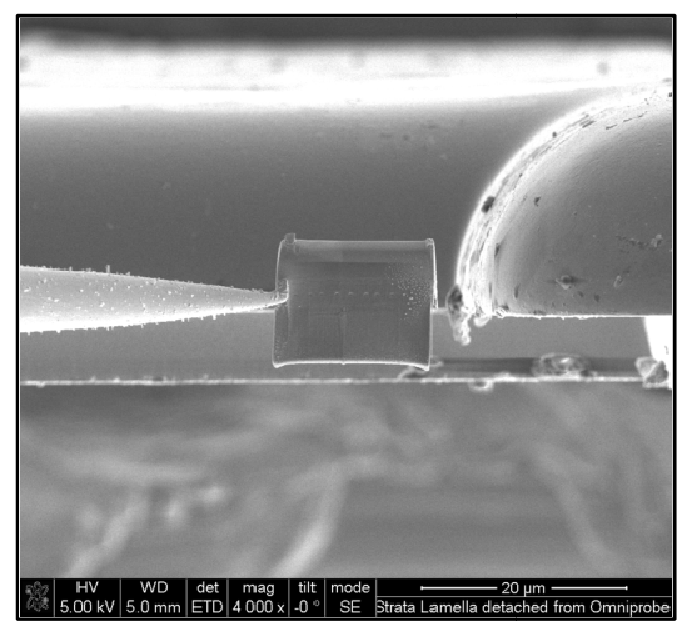

Figure 3. Specimen re-attached to micromanipulator after removal from the grid in preparation for Flip-2. Grid is at " 0 " " position.

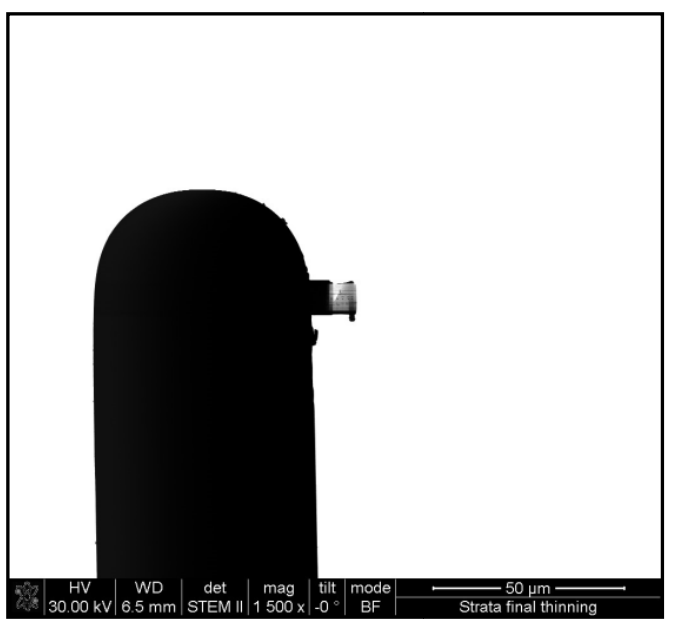

Figure 4. Low magnification STEM image of Twice Flipped specimen on grid that is ready for TEM imaging.

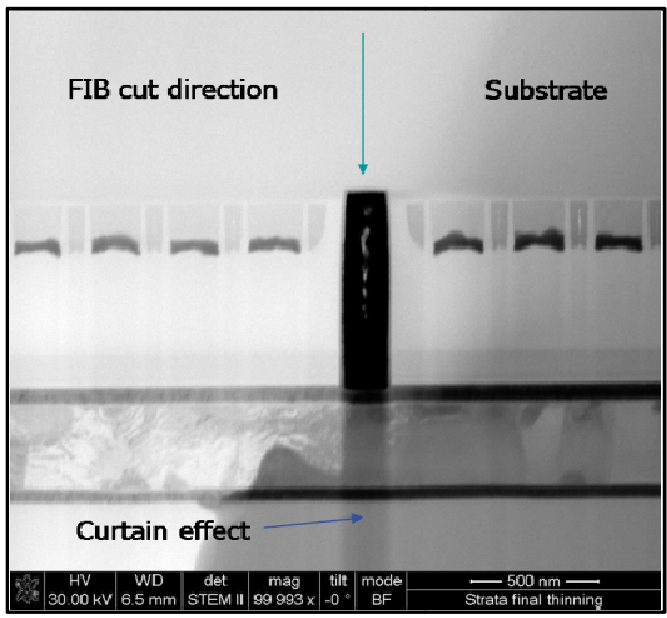

Figure 5. STEM image of Twice Flipped specimen at AOI.

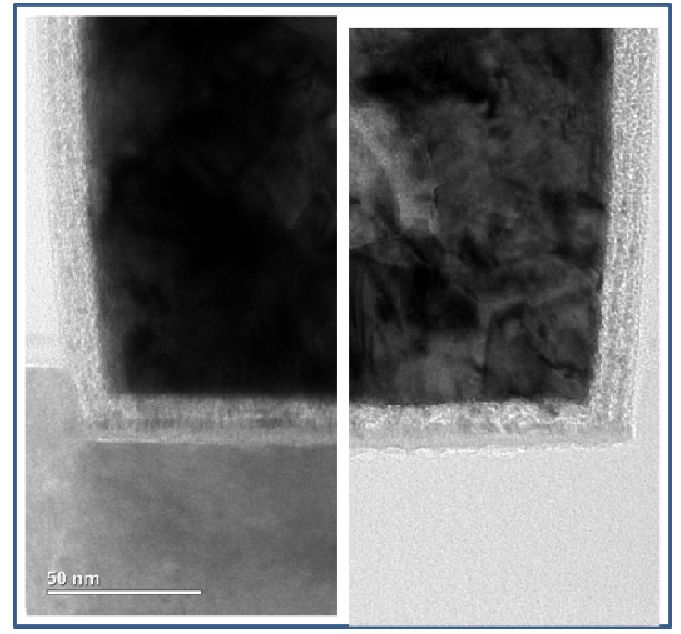

Figure 6. TEM micrographs comparing traditional approach (left) to the Twice Flipped specimen (right), showing a much more detail at the substrate interface and within bottom diffusion barrier layers. 